\title{
L'avalanche de Péclerey du 9 février 1999
}

\author{
The Péclerey avalanche of February 9th 1999 \\ par Christophe Ancey ${ }^{(1)}$, François Rapin ${ }^{(1)}$, Eric Martin (2), Cécile Coleou ${ }^{(2)}$, \\ Mohamed Naaim ${ }^{(1)}$, Gilles Brunot ${ }^{(3)}$
}

(1) Cemagref ; (2) Centre d'études de la neige, Météo-France ; (3) Centre départemental de Météorologie

In February 1999, an important avalanche activity took place in the Alps. Especially on February 9th, the Péclerey avalanche killed 12 persons in Montroc near Chamonix-Mont-Blanc, in a white zone (but not with no risk). This paper takes the final toll of this deasaster and questions arise concerning avalanche forecasting and prevention tools.

\section{INTRODUCTION}

En février 1999, l'activité avalancheuse dans les Alpes du Nord a connu une très grande ampleur et marquera la mémoire comme l'une des plus meurtrières depuis 50 ans. En l'espace de trois semaines, plusieurs dépressions frappent les Alpes, de la France à l'Autriche. Le 9 février, une avalanche tue 12 personnes dans des chalets à Montroc (France, Haute-Savoie, commune de Chamonix-Mont-Blanc); dans la même période, les vallées de Haute-Savoie, de Savoie et du Piémont (Italie) connaissent une activité avalancheuse très importante, puisque plusieurs avalanches atteignent les fonds de vallée, dépassant à plusieurs reprises les emprises historiques connues. Quelques jours plus tard, sous l'effet d'un second passage perturbé, c'est Evolène dans le Valais (Suisse) qui est touché. On dénombre 12 morts dans les habitations. Enfin, le 23 février, 38 personnes sont ensevelies dans deux stations du Tyrol, Galtür et Valzür (Autriche). Pour retrouver dans l'histoire récente une activité avalancheuse aussi meurtrière et couvrant une telle zone géographique, il faut remonter aux hivers 1970 (39 personnes tuées dans un chalet à Val-d'Isère), 1954 (143 personnes emportées en Autriche), 1951 ( 76 personnes tuées dans leurs habitations en Suisse). A l'échelle de la France, la crue avalancheuse du début février est comparable à celles de janvier 1981 et de février 1978, mais elle n'atteint pas l'ampleur et l'extension de la crue de février 1970 ou bien plus en arrière, des terribles hivers 1914 et 1923. L'objectif de cet article est de relater ce qui s'est passé le 9 février 1999 lorsque l'avalanche descendue de la montagne de Péclerey toucha le hameau de Montroc. Cette catastrophe amène également à se poser des questions quant aux outils actuels de prévision et de prévention des avalanches. Un rapide panorama sera dressé à la lumière des résultats de l'analyse sur l'avalanche de Péclerey.

\section{II — CONTEXTE GÉOGRAPHIQUE}

Montroc est un hameau de la commune de ChamonixMont-Blanc, situé au fond de la vallée de l'Arve, à une altitude moyenne de $1350 \mathrm{~m}$ (voir plan de situation de la figure 1). Il est situé à une dizaine de kilomètres environ du centre de Chamonix en direction de l'est, juste avant le village du Tour $(1450 \mathrm{~m})$. Au niveau de Montroc, la vallée de l'Arve est grosso modo d'orientation nord-est à sud-ouest. Le flanc orienté au sud (adret) est composé des pentes descendant de la tête du Chenavier (1927 m) et de la crête des Frettes. Le versant nord-ouest (ubac) est plus complexe. Le pied du versant est délimité par le lit de l'Arve (1391 m au droit de Montroc) tandis que sa limite supérieure est une ligne de crête très découpée, allant du Bec du Lachat $(2447 \mathrm{~m})$ au Bec de la Cluy $(2334 \mathrm{~m})$ et culminant à $2572 \mathrm{~m}$ (antécime du Bec Rouge inférieur). La pente moyenne du site est de $51 \%\left(27^{\circ}\right)$, ce qui n'est pas une valeur très forte pour un site avalancheux.

La figure 2 est une photographie prise quelques jours après la catastrophe ; elle montre une vue d'ensemble du site. Sur la figure 3 , nous avons reporté un profil en long du terrain naturel. Celui-ci est très irrégulier et il est utile de considérer quatre parties en liaison avec le fonctionnement avalancheux du site. Dans la partie supérieure du bassin-versant, la vaste conque concave formée par la ligne de crête allant du Bec du Lachat au Bec de la Cluy constitue la zone d'accumulation du site, c'est-à-dire la zone d'alimentation d'où peuvent partir les avalanches extrêmes du site et mobiliser le gros de leur volume. La limite inférieure de cette zone est plus difficile à cerner. Un peu arbitrairement, nous l'avons fixée à la courbe de niveau 2250. La zone d'accumulation couvre environ une dizaine d'hectares avec une pente moyenne de $74 \%\left(36^{\circ}\right)$. S'il faut interpréter cette valeur en termes de potentialité de départ d'avalanche, on peut dire qu'il s'agit d'une valeur plutôt moyenne. Sous la zone d'accumulation s'étale un vaste 


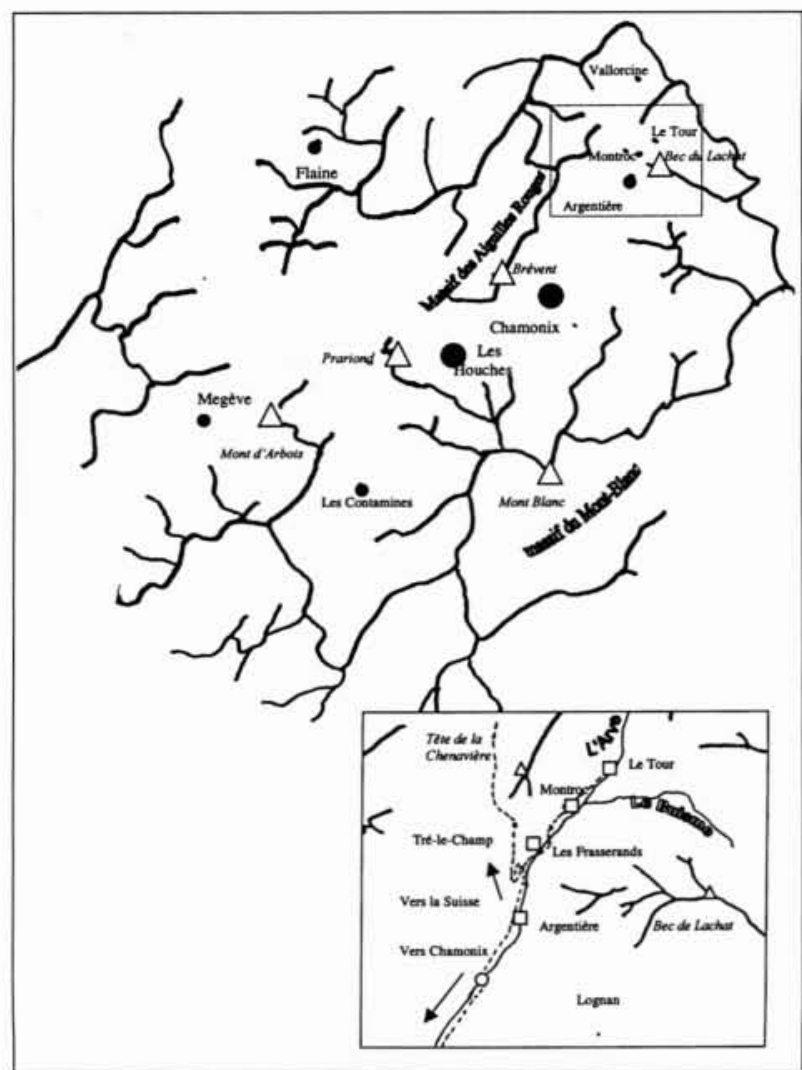

1. Carte de situation.

plan incliné entre 2250 et $1800 \mathrm{~m}$, désigné sous le nom de montagne de Péclerey (ancien alpage) et que nous appellerons ici zone de transit supérieure. Ses caractéristiques générales sont : un espace large assez peu confiné, composé d'un sol moutonné (glacier rocheux) couvert de pelouses alpines et d'éboulis grossiers, une pente ne dépassant pas $53 \%\left(28^{\circ}\right)$ et de valeur moyenne $36 \%\left(20^{\circ}\right)$ sur une grande distance $(700 \mathrm{~m})$. Une analyse spatiale de ce secteur en termes d'activité avalancheuse laisse penser qu'une avalanche partie de la zone d'accumulation doit avoir du mal à franchir la zone de transit supérieure, elle est probablement obligée de louvoyer entre les bosses du relief, à moins qu'elle ne prenne la direction du Tour (plus au nord). Comme cela est bien visible sur la figure 2, l'écoulement dans la direction nord-est est en effet favorisé par des pentes plus fortes et un relief moins vallonné. La courbe de niveau $1800 \mathrm{~m}$ délimite la frontière aval de la montagne de Péclerey : le terrain y accuse un changement brutal de pente d'environ $20^{\circ}$. Il existe donc entre la cote $1800 \mathrm{~m}$ et l'Arve $(1390 \mathrm{~m})$ un versant raide, que nous appellerons zone de transit inférieure. Le profil du terrain est grossièrement de forme parabolique, avec une pente diminuant régulièrement de 100 à $27 \%\left(45\right.$ à $\left.15^{\circ}\right)$. L'altitude et l'orientation sont favorables au développement d'une forêt, principalement composée

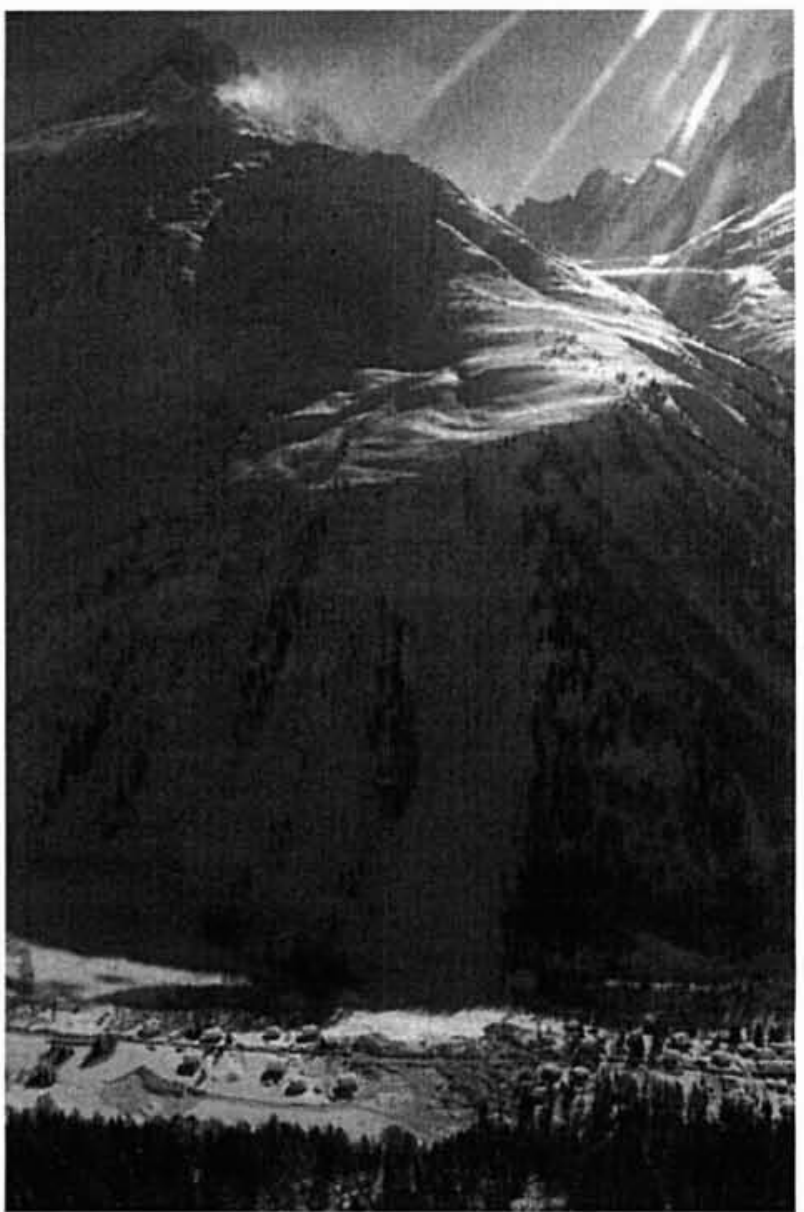

2. Photographie du site de Péclerey (cliché F. Rapin, Cemagref, du 12 février 1999).

de mélèzes, puis de sapins et de pins à crochets. Cette forêt peu dense est rayée de couloirs principalement colonisés par une végétation arbustive (aulnes nains). Ces couloirs dans la forêt sont les traces d'avalanches passées parties du Bec du Lachat ou bien résultent de coulées et d'avalanches qui freinent la reprise de la forêt. L'Arve passant en pied de versant, il n'y a pas à proprement parler de zone de dépôt sur l'ubac.

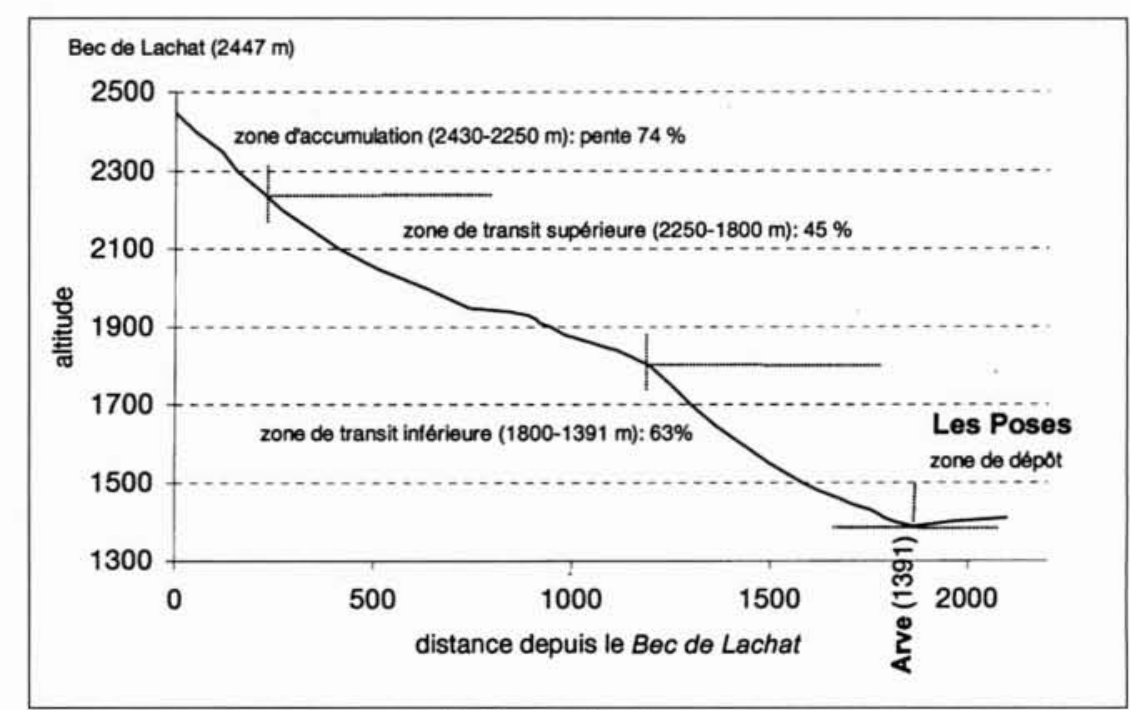

3. Coupe du site. 


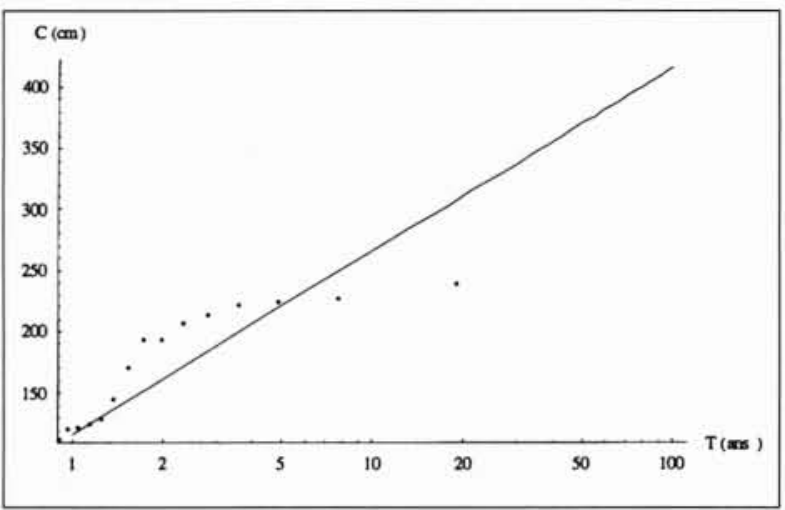

4. Distribution empirique des cumuls de neige $\mathrm{C}(\mathrm{en} \mathbf{c m})$ sur un épisode neigeux (de durée quelconque) en fonction de la période de retour $T$ (en ans) sur la station du Tour $(1470 \mathrm{~m})$. Le cumul sur un épisode est calculé comme étant la somme des cumuls journaliers entre le début et la fin de l'épisode neigeux. La durée des épisodes considérés est généralement de quelques jours. Nous avons sélectionné toutes les valeurs de cumul dépassant $80 \mathrm{~cm}$. En tout, nous avons trouvé 23 chutes de neige en 13 ans satisfaisant à $\mathbf{C}>80 \mathrm{~cm}$. La courbe continue représente la loi calée par la méthode du renouvellement (Poisson + exponentielle simple) : $C(T)=80+64,8 \log (1,77 \mathrm{~T})$. Le faible nombre de données est à l'origine de la faible adéquation entre courbe théorique calée et mesures. Avec un cumul de $225 \mathrm{~cm}$, l'épisode du $5-10 / 02 / 99$ a une période de retour de 5 ans.

Il faut au contraire imaginer que celle-ci s'étend sur l'adret, c'est-à-dire en rive droite de l'Arve sur le versant opposé à la montagne de Péclerey. Si cette configuration de site n'est pas la plus courante, elle n'est pas non plus exceptionnelle, surtout dans les vallées alpines où l'érosion glaciaire a donné des formes en $\mathrm{V}$ au début de fond de vallée. La zone de dépôt est donc ici considérée être en rive droite de l'Arve. Pour une avalanche, elle a une pente négative, car celle-ci est obligée de remonter l'adret. La limite inférieure de la zone de dépôt est imposée par le lit de l'Arve (1390 m environ) tandis que nous considérons que la limite forestière à $1420 \mathrm{~m}$ d'altitude donne une indication de sa limite supérieure. La pente de la zone d'arrêt est douce : de -5 à $-10 \%$ (de 3 à $6^{\circ}$ ). Le profil du terrain n'est pas très régulier. Un talus court et raide domine le lit majeur de l'Arve tandis qu'au-dessus de ce talus, la pente est plus douce et régulière. C'est à ce niveau, au lieu-dit les Poses, qu'ont été construits plusieurs chalets du hameau de Montroc. Cette zone résidentielle est par ailleurs traversée par la route reliant le Tour au cœur du village de Montroc (et à Chamonix).

\section{III - CONTEXTE CLIMATIQUE}

Le village du Tour est connu comme étant l'un des villages les plus enneigés de France. Un enneigement abondant y est favorisé compte tenu de la situation générale du massif du Mont-Blanc (vis-à-vis des dépressions de nord-ouest), la position en fond de vallée, et l'altitude élevée. Distant de $2 \mathrm{~km}$ et à une altitude sensiblement égale, le village de Montroc est bien représenté sur la plan climatologique par la station météorologique du Tour $(1470 \mathrm{~m})$, fonctionnant depuis I'hiver 1985/86. Quoique la période soit un peu courte pour estimer avec confiance une distribution des chutes de neige

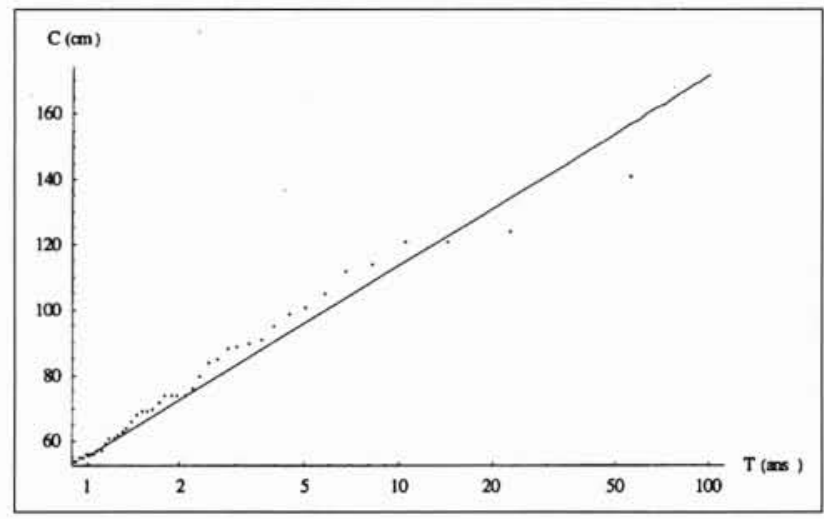

5. Distribution empirique des cumuls de neige $\mathbf{C}(\mathrm{en} \mathbf{~ c m})$ sur un épisode neigeux (de durée quelconque) en fonction de la période de retour $\mathbf{T}$ (en ans) sur la station de Chamonix $(1050 \mathrm{~m})$. Le cumul est calculé comme étant la somme des cumuls journaliers entre le début et la fin de l'épisode neigeux. La durée des épisodes considérés est généralement de quelques jours. Attention, les données sont issues du poste du CEDONIGLA entre 1959 et 1990 et du CDM depuis 1991. Nous avons sélectionné toutes les valeurs de cumul dépassant $50 \mathrm{~cm}$. En tout, nous avons trouvé 48 chutes de neige en 39 ans satisfaisant à $\mathrm{C}>50 \mathrm{~cm}$ ). la courbe continue représente la loi calée par la méthode du renouvellement (Poisson + exponentielle simple) : C $(\mathrm{T})=50+25,3 \log (1,23 \mathrm{~T})$. La loi satisfait au test de vraisemblance du $\chi^{2}$. Avec un cumul de $140 \mathrm{~cm}$, l'épisode du 6-10/02/99 a une période de retour de 35 ans.

extrêmes, le dépouillement des valeurs enregistrées donne quand même une tendance générale. Nous avons reporté sur la figure 4 la distribution des cumuls de neige (sur un épisode) en fonction de la fréquence. On notera que des cumuls supérieurs à $2 \mathrm{~m}$ ne sont pas exceptionnels sur le Tour. Nous avons reporté sur la figure 5 la distribution des chutes de neige pour Chamonix, dont le poste climatique (à $1050 \mathrm{~m}$ ) fonctionne depuis 1959. On notera une différence significative des cumuls entre les deux postes. La différence d'altitude de l'ordre de $400 \mathrm{~m}$ est bien sûr l'une des principales causes de cet écart, mais également le positionnement en fond de vallée (piégeant l'air froid) du Tour. Il est en effet fréquent que durant le passage d'une dépression, il neige sur le Tour alors qu'il pleut à Chamonix. Nous reportons dans les tableaux 1 et 2 les valeurs "records" de chutes de neige sur les deux postes météorologiques.

\section{IV — LES CONDITIONS NIVO-MÉTÉOROLOGIQUES PRÉCÉDANT L'AVALANCHE}

L'hiver 1998-99 commence avec de maigres chutes de neige sur les Alpes du Nord. A Noël, le manteau neigeux n'excède pas $30 \mathrm{~cm}$ dans les versants au nord à $1000 \mathrm{~m}$ d'altitude, accusant un déficit par rapport à la moyenne saisonnière. Il faut attendre la fin de janvier pour voir les premières chutes de neige significatives. Du 26 au 30 janvier, il tombe ainsi $150 \mathrm{~cm}$ de neige au Tour. Un beau temps froid et venté s'intercale avant l'arrivée de la dépression suivante. A partir du 5 février, un puissant flux de nord-ouest touche les Alpes. Il fait particulièrement froid, avec un isotherme $-10^{\circ} \mathrm{C}$ en moyenne voisin de $2500 \mathrm{~m}$. De grosses chutes de neige sont observées sur les Préalpes et dans les massifs intérieurs 
Eau : ressources, aménagements, environnement

\begin{tabular}{|ccc|}
\hline Cumul de la neige $(\mathrm{cm})$ & Date & Durée de l'épisode $(j)$ \\
\hline 205 & $25 / 02-04 / 03 / 89$ & 8 \\
\hline 212 & $23-28 / 03 / 88$ & 6 \\
\hline 220 & $11-17 / 01 / 86$ & 7 \\
\hline 223 & $16-23 / 12 / 86$ & 8 \\
\hline 225 & $6-11 / 02 / 99$ & 5 \\
\hline 238 & $5-13 / 02 / 88$ & 9 \\
\hline
\end{tabular}

Tableau 1. - Récapitulatif des chutes de neige sur le Tour depuis 1986 ayant dépassé $200 \mathrm{~cm}$ de cumul.

\begin{tabular}{|ccc|}
\hline Cumul de neige $(\mathbf{c m})$ & Date & Durée de l'épisode $(\boldsymbol{j})$ \\
\hline 100 & $21-26 / 12 / 68$ & 6 \\
\hline 104 & $11-18 / 02 / 85$ & 8 \\
\hline 111 & $19-22 / 12 / 92$ & 4 \\
\hline 113 & $17-22 / 02 / 63$ & 5 \\
\hline 120 & $04-12 / 01 / 68$ & 9 \\
\hline 123 & $15-21 / 01 / 81$ & 7 \\
\hline
\end{tabular}

Tableau 2. - Récapitulatif des chutes de neige sur Chamonix depuis 1959 ayant dépassé $100 \mathrm{~cm}$ de cumul.

\begin{tabular}{|c|c|c|c|}
\hline Modèle & Modèle VSG & Modèle AVAER & Modèle bi-couche \\
\hline Type d'avalanche décrit & Coulante & Aérosol & Tout type sec \\
\hline Type de mouvement & Unidimensionnel & Unidimensionnel & tridimensionnel \\
\hline Reprise & Non & Possible & Oui \\
\hline $\begin{array}{l}\text { Nombre de paramètres } \\
\text { mécaniques }\end{array}$ & 2 & 2 & 3 \\
\hline $\begin{array}{l}\text { Nombre de paramètres } \\
\text { géométriques }\end{array}$ & Profils en long, travers & Profils en long, travers & Modèle de terrain 3D \\
\hline $\begin{array}{l}\text { Nombre de conditions } \\
\text { initiales }\end{array}$ & 3 & 4 & 2 \\
\hline Calage des paramètres & Directives suisses & Expériences & Expériences \\
\hline Validation & Très importante (terrain) & Importante (laboratoire) & En cours \\
\hline Degré de confiance & Bon & Assez bon & \\
\hline Résultats fournis & $\begin{array}{l}\text { Vitesse, hauteur, pression, } \\
\text { distance d'arrêt }\end{array}$ & Géométrie, vitesse, pressions & $\begin{array}{l}\text { Géométrie, vitesse, } \\
\text { pression, concentration }\end{array}$ \\
\hline Remarques & $\begin{array}{l}\text { Donne des vitesses très } \\
\text { approximatives dans la zone } \\
\text { d'arrêt ; procédure spécifique } \\
\text { en cas de pentes négatives, } \\
\text { très sensible au paramètre de } \\
\text { frottement }(\mu)\end{array}$ & $\begin{array}{l}\text { Problème des conditions } \\
\text { initiales, pas de procédure } \\
\text { disponible en cas de pentes } \\
\text { négatives }\end{array}$ & $\begin{array}{l}\text { Modèle en cours de } \\
\text { développement }\end{array}$ \\
\hline
\end{tabular}

Tableau 3. - Récapitulatif des caractéristiques des modèles d'avalanche employés dans la reconstitution. 


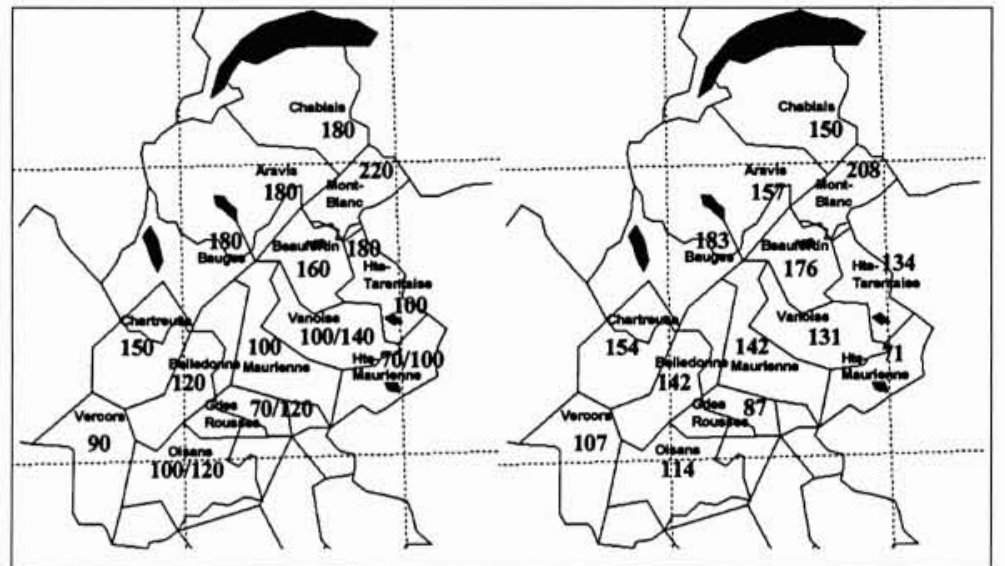

6. Cumul de neige fraîche sur 5 jours $(\mathrm{cm})$ du 5 au 10 février 1999 à gauche et lames d'eau cumulées sur 5 jours $(\mathrm{mm})$ à droite selon les massifs. Les valeurs de cumul sont issues du réseau d'observation tandis que les lames d'eau sont obtenues par SAFRAN (à $1800 \mathrm{~m}$ ).

des Alpes du Nord (Mont-Blanc, Beaufortain). Plus à l'intérieur et au sud, elles sont moins significatives. Quelques relevés : du 5 au 10, il tombe $225 \mathrm{~cm}$ au Tour (Vallée de l'Arve, massif du Mont-Blanc), $140 \mathrm{~cm}$ à Chamonix (Vallée de l'Arve, massif du Mont-Blanc), $170 \mathrm{~cm}$ à Megève (1070 m, massif du Mont-Blanc), $190 \mathrm{~cm}$ à Avoriaz (1780 m, Chablais), $186 \mathrm{~cm}$ à Arêches (1200 m, Beaufortain), $180 \mathrm{~cm}$ à la Rosière $(1900 \mathrm{~m}$, Tarentaise $), 154 \mathrm{~cm}$ à la Plagne $(1970 \mathrm{~m}$, Tarentaise), $137 \mathrm{~cm}$ aux Arcs (2040 m, Tarentaise), $113 \mathrm{~cm}$ aux Menuires (1800 m, Vanoise), $45 \mathrm{~cm}$ au col du Montgenèvre ( $1850 \mathrm{~m}$, Briançonnais), $145 \mathrm{~cm}$ au col de Porte (1360 m, Chartreuse). En moyenne, la lame d'eau estimée à $1800 \mathrm{~m}$ est de $208 \mathrm{~mm}$ dans le massif du Mont-Blanc, elle est proche de $150 \mathrm{~mm}$ sur les Préalpes du Nord, et descend au-dessous des $140 \mathrm{~mm}$ dans les massifs internes (cf. figure $\mathrm{n}^{\circ} 6$ ). Analysées à la lumière de leur période de retour, certaines chutes de neige ont un caractère remarquable. La période de retour du cumul de neige sur Chamonix du 5 au 10 février est ainsi estimée autour de 40 ans. Sur le Tour (et probablement Montroc), elle n'est que de 5 ans. Ces deux cas sont d'ailleurs assez représentatifs des stations respectivement de moyenne altitude et d'altitude. En revanche, si on analyse les chutes en termes de leur valeur en eau, la période de retour est inférieure à 5 ans, ce qui tend à prouver que, dans l'ensemble, l'écart trouvé dans l'estimation des périodes de retour d'un site à l'autre est principalement dû aux températures très froides accompagnant la dépression. Si les chutes de neige sur le massif du Mont-Blanc ne sont pas exception-

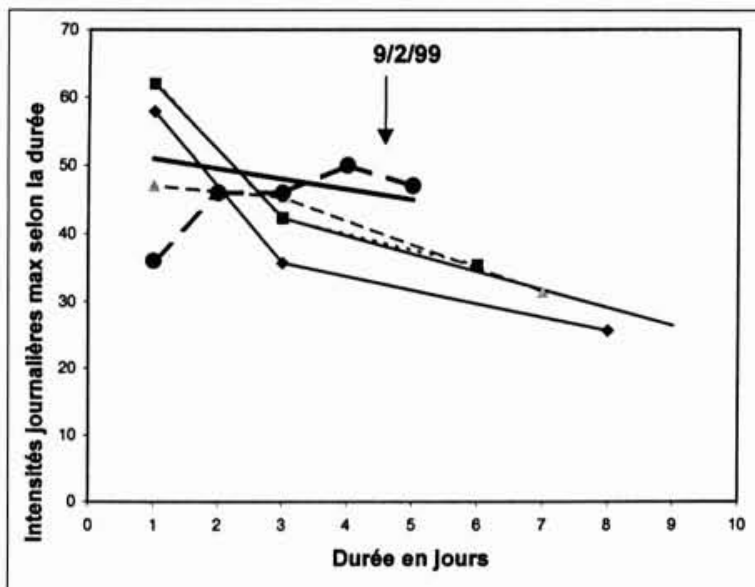

8. Résultat de la modélisation Crocus sur une pente Nord à 40 degrés, à $2400 \mathrm{~m}$, dans le massif du MontBlanc le 9 février à $6 \mathrm{~h}$. Sur la partie gauche du graphique, la courbe en pointillés est la température, en trait plein la résistance à l'enfoncement. Les deux flèches horizontales (très proches l'une de l'autre) indiquent les niveaux d'instabilité si un risque de départ d'avalanche a été diagnostiqué. Sur la partie droite, les types de grain de chaque couche sont représentés par des symboles (+ : neige fraîche, /particules reconnaissables, - grains fins, $\square$ grains à faces planes, $\bigcirc$ grains ronds). Les colonnes suivantes indiquent le diamètre des grains de neige (en $\mathrm{mm}$ ), la densité de la neige (en $\mathrm{kg} / \mathrm{m}^{3}$ ) ainsi que le rapport résistance au cisaillement contrainte (paramètre mécanique indiquant le caractère plus ou moins stable d'une couche de neige).
7. Distribution des intensités maximales de chutes de neige en fonction de la durée pour chacun des gros épisodes sur le Tour depuis 1986 et distribution des cumuls de neige journaliers pour l'épisode du 6 au 11 février 1999. Hormis la dernière courbe, il s'agit des maxima en un jour, trois jours, et de la moyenne sur la durée totale des intensités indépendamment de la chronologie de chaque chute. Au contraire la dernière courbe donne les hauteurs de neige en séquence dans la chronologie.

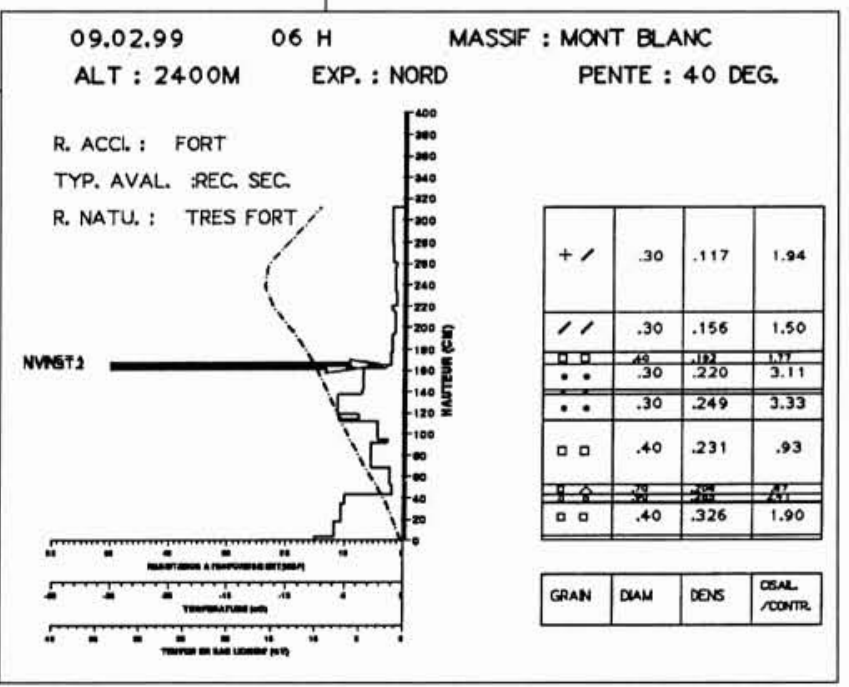


nelles en termes de cumul de neige, elles sont quand même associées à une caractéristique remarquable : elles ont été régulièrement soutenues comme le montre la figure 7 . Ainsi on observe que, sur le Tour, l'intensité de la chute de neige sur 1 jour n'arrive qu'en troisième position des intensités maximales journalières mesurées. En revanche, dès que l'on calcule l'intensité moyenne sur 3 jours ou plus, on note qu'elle reste à un niveau élevé. En général, comme l'illustre la figure 7, l'intensité moyenne sur plusieurs jours est significativement plus faible que l'intensité journalière maximale. Comme l'intensité des chutes de neige joue directement sur la stratification du manteau neigeux et indirectement sur ses métamorphoses, ce caractère particulier a vraisemblablement été un facteur propice à l'activité avalancheuse (outre les températures très froides favorisant une neige légère et sèche jusqu'à des altitudes assez basses).

S'il n'a pas été possible de procéder à un sondage du manteau neigeux au droit de la cassure sous le Bec de Lachat, il a été en revanche possible de bénéficier des résultats des sondages effectués à proximité et des simulations du manteau neigeux réalisées par la chaîne de logiciels SAFRAN/ CROCUS/ MEPRA utilisée par Météo-France dans le cadre de l'élaboration quotidienne du bulletin de risque d'avalanches (BRA). Dans la figure 8, nous avons tracé un sondage par battage typique simulé par SAFRAN. Ce résultat de simulation est une approximation de l'état réel du manteau neigeux dans la zone de départ de l'avalanche (en particulier les phénomènes locaux de transport de neige par le vent ne sont pas pris en compte). Les grandes caractéristiques du profil simulé doivent s'y retrouver, notamment :

- une grande quantité de neige récente froide et de faible cohésion, plus d'1,5 m d'épaisseur en tenant compte des chutes de neige de la matinée du 9 février :

- cette neige a une densité faible, voisine de $110 \mathrm{~kg} / \mathrm{m}^{3}$, un peu plus dans la partie inférieure à cause du tassement ainsi que dans la partie supérieure avec le radoucissement qui s'est opéré dans la matinée ;

- une couche fragile est probablement présente à la base de cette neige récente. En effet, même si des vents violents ont soufflé du 30 janvier au $1^{\text {er }}$ février, il est possible que toute la neige récente de l'épisode de fin janvier n'ait pas été arrachée dans cette pente et que la neige de surface ait pu se transformer en grains anguleux (grains à faces planes) après cette période ;

- des couches profondes constituées en grande partie de faces planes ou gobelets.

Les conditions nivo-météorologiques ont été jugées comme très critiques par les services départementaux de Météo-France. Dès le dimanche 7 , un bulletin d'alerte est émis par le point focal de Météo-France à Saint-Martind'Hères en direction des autorités concernées et de la presse. Il prévient que des avalanches "pourront prendre une extension importante et éventuellement menacer des voies de communication ou diverses infrastructures parfois même dans des secteurs d'assez basse altitude". Le risque est confirmé par de nouveaux bulletins d'alerte le lundi 8 et le mardi 9 février : "on peut s'attendre à une aggravation de la situation, des avalanches de grande ampleur et à trajectoire parfois inhabituelle sont à redouter". Selon l'échelle de risque d'avalanche comportant 5 niveaux, le risque a été annoncé comme très fort dès l'après-midi du $5(5 / 5)$ et jusqu'au jeudi 11 février (où il repassa à 4/5).
La situation avalancheuse devint rapidement critique dès le dimanche 7. Elle atteint son paroxysme le mardi 9 février. Ainsi, dans la vallée de Chamonix, ce sont 14 avalanches majeures qui ont touché la vallée de Chamonix. Sur ces 14 avalanches, 6 eurent des emprises supérieures à celles connues (telles que reportées sur la carte de localisation probable des avalanches de Chamonix dans son édition de 1991). L'avalanche de Montroc eut lieu durant cette journée, vers $14 \mathrm{~h} 40$.

\section{DESCRIPTIF DE L'AVALANCHE DE PÉCLEREY DU 9 FÉVRIER 1999}

\section{- 5.1 Méthodologie de reconstitution}

Les connaissances sur des phénomènes naturels aussi complexes que les avalanches sont encore trop imparfaites pour qu'on puisse, même a posteriori, spécifier les causes et le déroulement d'un événement avec une absolue certitude. Pour arriver à une reconstitution la plus cohérente possible de l'avalanche du 9 février 1999 sur Montroc, nous avons recoupé toutes les sources d'information à notre disposition :

- observations de terrain : elles permettent d'une part d'appréhender le fonctionnement général du site (cf. \$2), d'autre part de recueillir des indices du passage de l'avalanche du 9 février 1999 (dégâts, emprise). L'analyse des photographies aériennes permet également de se faire une idée générale sur la direction des flux et l'influence du relief. Plusieurs visites de terrain ont été réalisées au cours de I'hiver et du printemps ; chacune d'elles a amené des éléments intéressants et offert un éclairage différent ;

- témoignages : même si ceux-ci sont assez maigres dans le cas présent, ils permettent d'apporter des indications précieuses sur ce qui a pu se passer dans la zone d'arrêt. Le plus souvent, ce sont des récits lus dans la presse et il n'a été possible ni de questionner tous les témoins (notamment les victimes survivantes), ni d'accéder aux constats de gendarmerie ; - résultats de modèles de calcul : à l'heure actuelle, il existe un nombre important de modèles de calcul. Parmi les plus usités actuellement en France, on peut citer les modèles empiriques, dont l'emploi est courant en ingénierie et les modèles de simulation, qui sont encore en cours de développement et commencent à être utilisés dans des expertises pointues. C'est principalement l'échelle d'espace considérée pour les calculs qui les distingue :

- les modèles empiriques se fondent sur des équations décrivant le mouvement global de l'avalanche : en général, les équations auxquelles on aboutit se résolvent facilement. Nous avons utilisé deux modèles empiriques de calcul des avalanches : pour les avalanches coulantes : le modèle de Voellmy. Nous avons utilisé la variante dite VSG (Voellmy-Salm-Gubler) datée de 1991 du modèle de Voellmy. Pour les avalanches en aérosol : nous avons utilisé le modèle AVAER de Béghin.

- les modèles de simulation sont basés sur un jeu d'équations locales de conservation, issues de la mécanique des milieux continus ; l'intégration de ces équations est nécessairement numérique, ce qui est très gourmand en temps de calcul. Par exemple, une simulation de l'avalanche de Montroc à l'aide du modèle bi-couche développé au Cemagref requiert actuellement 4 jours de calcul contre quelques secondes pour un modèle empirique. 
Une différence également notable entre modèles empiriques et de simulation est que les premiers travaillent en prédétermination, c'est-à-dire que, hormis la hauteur de neige, tous les paramètres sont choisis selon des directives ou parmi des gammes usuellement employées en ingénierie : ces valeurs ont été déterminées au fil des années par calage sur un grand nombre d'événements (naturels ou reproduits en laboratoire). Au contraire, les modèles de simulation (développés au Cemagref) ne disposent pas encore d'une base suffisante d'événements ayant pu servir à leur validation ou au calage des paramètres. Les paramètres ont été calés à partir d'expériences sur maquette réalisées sur de la neige sèche au col du Lac Blanc. Si les modèles de simulation reposent sur un niveau de description bien plus fin que celui des modèles empiriques, ils ne constituent pas encore un outil qui permette de trancher sur la nature des phénomènes physiques dans une avalanche, car ils reposent sur une description physique assez simplifiée. La situation est donc différente de l'hydraulique classique, où les outils numériques sont aujourd hui à même de reconstituer assez fidèlement une crue. A défaut de lever l'incertitude sur le déroulement de l'avalanche, ces modèles permettent quand même d'aboutir à une approximation des caractéristiques de l'avalanche (vitesse, hauteur, pression). Le tableau 3 récapitule les principales caractéristiques des modèles employés.

\subsection{Le départ de l'avalanche}

Vers $14 \mathrm{~h} 40$, la majeure partie de la zone d'accumulation (plus principalement la partie centrale sous le Bec du Lachat) décroche naturellement vraisemblablement sous l'effet de son propre poids. Toute la couche de neige résultant des dernières chutes de neige est mobilisée. La cassure est haute de 1 à $2 \mathrm{~m}$ et s'étend sur une largeur d'à peu près $250 \mathrm{~m}$ à environ $50 \mathrm{~m}$ sous l'arête rocheuse allant du Bec de Lachat au Bec de la Cluy. La figure 2 montre la zone de départ. Lavalanche mobilise environ $90000 \mathrm{~m}^{3}$ de neige récente, sèche et relativement légère (densité inférieure à $0.15)$. Assez rapidement, la vitesse acquise par l'avalanche est relativement importante, de l'ordre de $25 \mathrm{~m} / \mathrm{s}$.

\section{- 5.3 Le parcours de l'avalanche}

Dans la partie supérieure de la zone de transit, l'avalanche s'essouffle un peu. La pente insuffisante et le relief moutonné ne favorisent pas son écoulement. Toutefois, elle parvient à franchir cette zone en s'étalant assez largement, notamment en direction du nord. Le frottement sur le man-

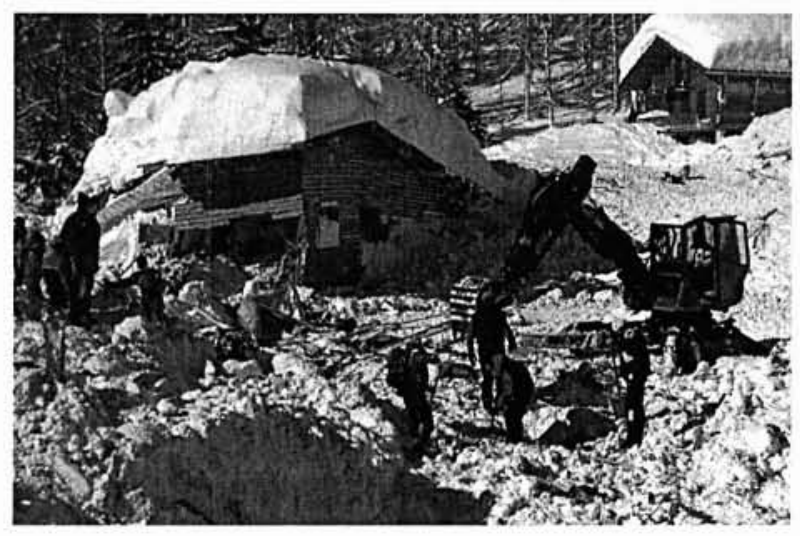

9. Zone d'arrêt de l'avalanche (cliché F. Rapin, Cemagref, pris le 10 février 1999). teau neigeux est limité compte tenu de la faible consistance de la neige et, profitant de quelques accélérations locales, l'avalanche arrive à garder une vitesse suffisante (de l'ordre de $20 \mathrm{~m} / \mathrm{s}$ en moyenne) et à reprendre encore de la neige au sol (de l'ordre de $60000 \mathrm{~m}^{3}$ ). La largeur de l'écoulement atteint $400 \mathrm{~m}$. L'écoulement est relativement dense et proche du sol, sous forme d'une nappe de 2 à $4 \mathrm{~m}$ d'épaisseur mobilisant de la neige sans cohésion. Un petit panache le surmonte mais il n'a aucun rôle dynamique particulier.

Lorsque l'avalanche atteint la cote $1800 \mathrm{~m}$, elle subit plusieurs transformations. Premièrement, le relief favorise la séparation continue de l'avalanche en grosso modo deux branches, l'une en direction du Tour, l'autre en direction de Montroc. Cette dernière est la plus grosse, avec un volume de l'ordre de $160000 \mathrm{~m}^{3}$. Deuxièmement, la rupture de pente sous la courbe de niveau $1800 \mathrm{~m}$ donne une nouvelle impulsion à l'avalanche. Pour le couloir qui nous intéresse. le changement de dynamique de l'avalanche est important. D'une part, la rupture de pente induit une aération de l'écoulement (forte incorporation d'air), ce qui génère un aérosol. D'autre part, il permet une accélération notable de tous les flux en mouvement, ce qui limite l'élargissement et maintient une concentration assez forte. L'avalanche atteint une vitesse moyenne de l'ordre de $40 \pm 10 \mathrm{~m} / \mathrm{s}$. La hauteur de la partic dense de l'avalanche est comprise entre 2 et $5 \mathrm{~m}$ et sa largeur vaut approximativement $150 \mathrm{~m}$. De plus, compte tenu des fortes pentes dans la partie inférieure de la zone de transit, l'avalanche reprend la presque totalité du manteau neigeux. A l'arrivée sur l'Arve. l'avalanche s'élargit nettement (de l'ordre de $200 \mathrm{~m}$ ) sous l'effet de la réduction de pente mais garde une vitesse encore importante (supérieure à $25 \mathrm{~m} / \mathrm{s}$ ) et une densité encore faible (inférieure à 0.15 ) .

Profitant de son inertie, le corps dense de l'avalanche remonte facilement les premiers mètres de l'adret. Il est précédé de quelques secondes par la partie en aérosol. Cette partie composée de neige en suspension dans l'air se déplace un peu plus rapidement, elle s'étend sur des hauteurs importantes (supérieures à $25 \mathrm{~m}$ ) et touche en premier les habitations. Elle n'a toutefois aucun rôle destructeur : elle correspond juste à l'effet de souffle ressenti par la plupart des riverains. En revanche, le corps dense de l'avalanche a une force et une inertie bien supérieures. Haut d'environ $3 \mathrm{~m}$, il exerce une pression supérieure à $100 \mathrm{kPa}$ sur les rezde-chaussée des premiers chalets rencontrés (majoritairement en bois), qui ne peuvent résister et sont littéralement laminés. Au fur et à mesure de la remontée sur l'adret, l'avalanche a dû progressivement perdre de sa vigueur, notamment à l'amont de la route. La photographie de la figure 9 prise durant les opérations de secours montre la zone d'arrêt de l'avalanche couverte des débris des habitations et le premier étage d'un chalet transporté sur plusieurs mètres par l'avalanche après qu'elle a eu détruit le rez-dechaussée. En tout, ce sont vingt chalets, qui sont concernés : 14 sont détruits et 6 sont endommagés (avec des dégâts matériels plus ou moins importants).

Le dépôt a été estimé à $250 \mathrm{~m}$ de long, $150 \mathrm{~m}$ de large, et environ 4 mètres d'épaisseur en moyenne. Cela conduit à un volume voisin de $150000 \mathrm{~m}^{3}$ avec une masse volumique moyenne de $250 \mathrm{~kg} / \mathrm{m}^{3}$. À l'aval de la route (vers le sudest), l'épaisseur du dépôt s'évalue entre 4 et $8 \mathrm{~m}$ : les premiers niveaux des habitations ont tous été détruits et recouverts. Cette épaisseur s'atténue progressivement et assez insensiblement vers l'amont passant de 2 à $5 \mathrm{~m}$. 


\section{VI — COMMENTAIRES ET CONCLUSION}

L'avalanche du 9 février 1999 partant du Bec de Lachat est un phénomène complexe et peu ordinaire par ses caractéristiques. Il faut se garder de vouloir la réduire à un type bien précis d'avalanche. En effet, on estime que, d'une part. elle a dû présenter des caractéristiques très variables selon les tronçons (en termes de vitesses et de forme d'écoulement). Il s'est vraisemblablement agi d'une avalanche très rapide (plusieurs dizaines de mètres par seconde), de neige sèche et très fluide, en moyenne pas très dense (moins de $200 \mathrm{~kg} / \mathrm{m}^{3}$ ) avec un centre de gravité proche du sol, et une hauteur d'écoulement avec ce niveau de densité assez moyenne (moins de $5 \mathrm{~m}$ ), surmontée par un aérosol qui n'a vraisemblablement pas causé de dégâts significatifs. Elle s'est également caractérisée par la brutalité de son impact avec les bâtiments et une distance d'arrêt peu commune en regard de phénomènes comparables connus. Il nous semble que plusieurs facteurs ont contribué à l'ampleur de cette avalanche. Tout d'abord, les chutes de neige accompagnées d'une température très froide ont permis l'établissement d'une importante couche de neige sèche facilement mobilisable tout le long du trajet de l'avalanche. La présence de neige sans cohésion datant du début de saison a pu encore favoriser la reprise de neige en place par l'avalanche dans les parties raides du site. Ensuite, la neige mobilisée sèche, très froide devait présenter un caractère extrêmement fluide une fois mise en mouvement. Enfin, la configuration du site, avec cette rupture de pente marquée vers $1800 \mathrm{~m}$, a donné une nouvelle impulsion à l'avalanche et a généré un aérosol.

Il a été montré plus haut que la période de retour des chutes de neige en termes de cumul du début février 1999 est de l'ordre de 5 ans. Naturellement, cette estimation ne tient compte que du cumul et non d'autres paramètres comme les températures très froides. Même s'il était possible d'intégrer ce type de paramètres dans l'estimation de la période de retour, cela n'affecterait pas notablement le résultat, surtout pour les domaines de haute montagne. Durant ces vingt dernières années, plusieurs chutes de neige ont eu des caractéristiques similaires ou supérieures à celle de 1999. Citons janvier 1981, janvier 1986, février 1988, mars 1989, février 1990. Plus remarquable est le fossé entre la période de retour des précipitations (relativement faible) et le caractère exceptionnel des dégâts occasionnés par l'avalanche du 9 février 1999. En l'état actuel des connaissances, il est difficile d'indiquer avec finesse la période de retour de l'avalanche. Tout d'abord parce que l'information historique manque, qu'elle est lacunaire, et qu'elle n'est pas sûre. Ainsi, si plusieurs sources mentionnent des avalanches dans le couloir de Péclerey, il ne nous a pas été possible de les recouper. Par exemple, un témoignage écrit mentionne une avalanche en 1843, mais il n'est pas certain que l'auteur parle de l'actuel couloir de Péclerey (cinq autres couloirs jouxtent celui de Péclerey) quand on examine le plan cadastral sarde pour repérer les lieux-dits cités. De même, le fichier de l'enquête permanente des avalanches (EPA) relate des événements en 1908, 1928, 1931, mais des erreurs manifestes sur les altitudes ne permettent pas d'exploiter pleinement les renseignements. Enfin un témoignage écrit daté de 1973 mentionne une avalanche en 1945 atteignant la route d'accès au Tour, mais il a été controversé par d'autres. Toujours est-il qu'en accordant crédit à tous ces témoignages, cela tend à prouver qu'en termes d'occurrence au-delà de l'Arve, l'événement du
9 février 1999 est au moins de fréquence pluri-décennale. Pour tenter d'aller un peu plus loin dans l'estimation de la période de retour, nous avons utilisé des simulations de Monte-Carlo sur la base du modèle de Voellmy pour reconstituer des chroniques d'avalanche : en tirant au hasard une épaisseur de neige au départ et un coefficient de frottement (tout en respectant les lois de distribution statistique estimées pour ces deux paramètres), nous avons fait tourner le modèle et enregistré les différentes caractéristiques de l'avalanche. Ainsi, après avoir recueilli plus de 500 événements fictifs, nous sommes arrivés à des périodes de retour comprises entre 150 et 300 ans selon la variable choisie pour faire la statistique (énergie, puissance d'impact, cote atteinte, etc.). Ces investigations sur l'estimation de la période de retour sont riches d'enseignements. Elles montrent notamment que localement, une situation avalancheuse catastrophique (mettons centennale) peut potentiellement se produire plusieurs fois par siècle si l'on ne considère que le cumul de neige fraîche comme principal ingrédient du déclenchement d'avalanche. Cela est cohérent avec la fréquence d'occurrence d'avalanches touchant des habitations dans les Alpes françaises, qui est de quelques années (dans l'histoire récente : 1970, 1978, 1981, 1988, 1990, 1995).

Montroc est typique des catastrophes d'avalanche survenues ces dernières années. Tout d'abord du point de vue météorologique, le scénario est souvent le même : succession de dépressions océaniques rapides dans un flux général de nord-ouest à ouest. Ce scénario est bien connu et les services départementaux de Météo-France ont correctement prédit le niveau de risque induit par le passage perturbé de février 1999. En termes de dégâts aux habitations, on notera que les dernières catastrophes ont principalement touché des secteurs à l'activité avalancheuse faible, voire réputée inexistante. C'est le cas de secteurs habités depuis fort longtemps comme Saint-Etienne-de-Cuines (Maurienne) et de La Morte (Isère) en janvier 1981, sans parler des domaines d'altitude (les Brévières-Tignes en 1990). Cela pose naturellement la question de savoir si nous sommes en mesure d'éviter pareilles catastrophes dans le futur. La réponse est en demi-teinte. En l'état actuel de la procédure, la réponse serait plutôt négative. En effet, dans l'esprit des plans de zonage actuels (plan de prévention des risques), est retenue principalement l'idée de se prémunir contre des risques avérés, dont on a une trace historique. Evidemment, en caricaturant, une telle logique conduit à l'implication : pas d'événement historique connu $\Rightarrow$ pas de danger. On pourrait imaginer d'autres procédures, par exemple une procédure qui couplerait analyse rétrospective (l'histoire du site) et prospective (le danger potentiel sur le site). Cette analyse spatiale du risque ne poserait pas beaucoup de difficultés dans la mesure où l'on peut se faire une image grossière de l'activité potentielle d'un site à travers quelques paramètres simples tels que pente, relief, orientation, etc. On peut même imaginer que l'utilisation de règles simples dans des systèmes d'information géographique (SIG) permettrait d'automatiser l'analyse spatiale. Ce faisant, on aurait aussi quelques surprises d'une part car il y aurait une extension significative des zones potentiellement avalancheuses, d'autre part car cette extension pourrait concerner des secteurs sans avalanche observée depuis des siècles. Cela pourrait prêter à sourire, mais souvenons-nous : il y a vingt ans, qui aurait prédit que Saint-Etienne-de-Cuines en fond de vallée (800 m d'altitude), protégé par une épaisse forêt sur 
$1000 \mathrm{~m}$ de dénivelée, puisse être touché par une avalanche partie d'une croupe à $2100 \mathrm{~m}$ d'altitude ? L'analyse spatiale du risque doit donc nécessairement être couplée à une analyse fréquentielle de l'aléa : sur un secteur potentiellement avalancheux, quelle est la fréquence d'occurrence d'un événement ? Pour répondre à cette question, on peut utiliser la chronique des événements passés dans la mesure où elle existe et est suffisamment documentée. Dans bien des secteurs vierges d'aménagement, jusqu'à une époque récente, cela n'est pas possible et il faut envisager d'autres méthodes. Par exemple, on peut utiliser une approche statistique : comme on l'a vu précédemment, il n'y a pas à proprement parler égalité entre périodes de retour d'une chute de neige et d'une avalanche, mais il y a peut-être une relation plus ou moins simple. Celle-ci peut être recherchée régionalement en cherchant les corrélations entre caractéristiques d'une activité avalancheuse et paramètres nivo-météorologiques. Une autre voie, plus déterministe, consisterait comme précédemment à recourir à une simulation de type Monte-Carlo pour créer une chronologie fictive d'avalanches sur un couloir donné. En bref, l'amélioration de la prévention passe nécessairement par une révision profonde des méthodes utilisées pour diagnostiquer l'aléa. A notre avis, cela demande non pas de se fonder sur le plus gros événement historique connu mais de coupler une analyse spatiale et fréquentielle du risque, puis de fixer un niveau d'aléa (fréquence centennale par exemple). Soulignons qu'à l'heure actuelle, si l'analyse spatiale est facilement réalisable (c'est grosso modo ce qui est fait dans les cartes de localisation probable des avalanches à travers la photo-interprétation), l'analyse fréquentielle en est à ses balbutiements. Le seul problème de la définition d'une période de retour d'une avalanche (ou d'un autre aléa gravitaire) n'a pas reçu de réponse à notre connaissance.

Un autre aspect soulevé par l'avalanche de Péclerey concerne le zonage. Un grand bruit, dans la presse notamment, a été fait sur le fait que des chalets situés en zone blanche ont été détruits. Implicitement en France, dans le grand public mais aussi chez beaucoup de responsables et techniciens, la zone blanche est synonyme de zone sans risque. Cette tautologie est dangereuse. Elle induit d'une part que le risque nul existe et d'autre part, l'appréciation du risque nul étant pour le moins subjective, elle laisse tout le poids de la décision au technicien en charge du zonage. Sa responsabilité pourrait être alors vue comme écrasante en cas d'accident, car il aurait commis une erreur, voire une faute. Dans ce cas, à terme, quelle autre option pourra-t-il rester aux personnes réalisant un zonage si ce n'est de diminuer très significativement les limites des zones blanches sans base objective autre qu'un légitime soin à s'éviter d'éventuels ennuis judiciaires ? La procédure actuelle de zonage fondée sur l'appréciation des événements historiques est de plus injuste dans son principe. On note ainsi des différences significatives d'extension du zonage sur un secteur selon qu'il est aménagé depuis une longue période ou non. Cela conduit nécessairement à des perspectives de développement qui peuvent parfois être très différentes d'une commune à une autre. Il paraît donc largement préférable de définir la zone blanche sur des bases plus objectives, notamment en s'aidant d'une analyse couplée spatiale et temporelle comme nous l'avons évoqué plus haut. Par exemple, en Suisse, la zone blanche est définie comme la zone hors de l'extension des avalanches tri-centennales.

Enfin, une confusion largement présente dans la tête de nos concitoyens concerne la prévision d'une avalanche. Dans une grande majorité de cas, les services de MétéoFrance sont capables de prévoir la mise en place d'une activité avalancheuse et d'en préciser les caractéristiques générales. Il n'est en revanche pas possible actuellement de prédire quel site sera touché en particulier. Dans le cas présent, si la crue avalancheuse dans la vallée de Chamonix était prévisible, l'avalanche de Péclerey ne l'était pas. Reste à savoir si, dans l'avenir, nous serons capables de progresser en affinant la prévision en indiquant les zones à activité avalancheuse certaine ou probable. Ce grand chantier est en cours au CEN et au Cemagref, avec notamment un projet d'une liaison complète entre prévision des paramètres nivométéorologiques (l'actuel ensemble SAFRAN/ CROCUS/ MEPRA), calcul du développement de l'instabilité d'un manteau neigeux, et calcul de sa mise en mouvement.

\section{Remerciements}

Cet article est tiré du travail d'expertise qui nous a été confié par la préfecture de Haute-Savoie à la suite de la catastrophe de Montroc. Ce travail a bénéficié de l'aide de plusieurs organismes et personnes. Nous tenons plus particulièrement à remercier pour leur précieuse aide : la mairie de Chamonix-Mont-Blanc et notamment Jean-Marc Bonino, le peloton de Gendarmerie de Haute Montagne de Chamonix en la personne de l'adjudant-chef Trousselier, l'association des Amis du Vieux Chamonix, notamment René Simond, le fond Payot des Archives départementales de la Haute-Savoie et plus particulièrement madame Brigitte Pélissier, Messieurs René Arpin, Charles Gardelle, Peter Borggaard, René Ducroz, Claude Charlier, Madame Anne-Marie Granet Abysset. Au sein du Cemagref, plusieurs personnes ont apporté leur concours. Merci plus particulièrement à : Maurice Meunier, Florence Guirado, Sébastien Escande, Dominique Strazzeri, François Lacroix, Laurent Bélanger, et Philippe Revol. 